\title{
INVESTIGACIÓN
}

\section{Estudio comparativo de la obtención de extractos crudos y semipurificados de lipasa de salvado de arroz}

\author{
Por A. Bella Cruz y D. Barrera-Arellano \\ Laboratório de Óleos e Gorduras; DTA/FEA/UNICAMP Caixa Postal 6091, 13.083-970 \\ Campinas SP, Brasil. E-mail: barrera @obelix.unicamp.br
}

\section{RESUMEN}

Estudio comparativo de la obtención de extractos crudos y semi-purificados de lipasa de salvado de arroz.

Los extractos crudos de lipasas de salvado de arroz desengrasado fueron obtenidos con tres diferentes soluciones de extracción: 1 - $\mathrm{CaCl}_{2} 0.01 \mathrm{M} ; 2$ - Sacarosa 0.6M, $\mathrm{KCl} 10 \mathrm{mM}, \mathrm{MgCl}_{2} 1 \mathrm{mM}$, Ditiotreitol $2 \mathrm{mM} ; 3$ - Sacarosa $0.4 \mathrm{M}$, Ditiotreitol $2 \mathrm{mM}$. Las variedades estudiadas $\mathrm{pH}(6.0,7.0,8.0)$ y tiempo de extracción $(1 \mathrm{~h}, 2 \mathrm{~h}, 3 \mathrm{~h})$. La temperatura de extracción fue de $4{ }^{\circ} \mathrm{C}$. Los extractos obtenidos presentaron diferentes actividades de lipasa siendo el extracto crudo obtenido con $\mathrm{CaCl}_{2} 0.01 \mathrm{M}$ el que mostró la mayor actividad específica (12. $1 \mathrm{mU} / \mathrm{mg})$. La purificación parcial de este extracto crudo con acetona y sulfato amónico resultó en fracciones con actividad específica de $50.7 \mathrm{mU} / \mathrm{mg}$ y $63.5 \mathrm{mU} / \mathrm{mg}$ respectivamente.

PALABRAS-CLAVE: Enzima - Extracción - Lipasa - Purificación - Salvado de arroz.

\section{SUMMARY}

Comparative study of the obtention of crude and semi-purified lipase extracts from rice bran.

Crude lipase extracts from defatted rice bran were obtained using three different extraction solutions: $1-\mathrm{CaCl}_{2} 0.01 \mathrm{M}$; 2 - Sacarose $0.6 \mathrm{M}, \mathrm{KCl} 10 \mathrm{mM}, \mathrm{MgCl}_{2} 1 \mathrm{mM}$, Ditiotreitol $2 \mathrm{mM}$ and 3 - Sacarose 0.4M, Ditiotreitol $2 \mathrm{mM}$. Factors (variables) studied were $\mathrm{pH}(6.0,7.0,8.0)$ and extraction time $(1 \mathrm{~h}, 2 \mathrm{~h}, 3 \mathrm{~h})$. Extraction temperature was $4{ }^{\circ} \mathrm{C}$. The extracts obtained presented different lipase activities, and the crude extract obtained with $\mathrm{CaCl}_{2} 0.01 \mathrm{M}$ was the one with the highest specific activity $(12.1 \mathrm{mU} / \mathrm{mg})$. Partial purification of this crude extract with acetone and ammonium sulfate resulted in fractions with specific activities of $50.7 \mathrm{mU} / \mathrm{mg}$ and $63.5 \mathrm{mU} / \mathrm{mg}$, respectively.

KEY-WORDS: Enzyme - Extraction - Lipase - Purification Rice bran.

\section{INTRODUCCIÓN}

Las lipasas (triacilglicerol acil-hidrolasa, EC 3.1.1.3) son enzimas que catalizan la hidrólisis de triacilgliceroles liberando ácidos grasos y glicerol (IUPAC-IUB, 1979).
Sin embargo en presencia de solventes orgánicos o en sistemas anhidros, las lipasas pueden presentar un comportamiento totalmente diferente, catalizando reacciones de esterificación y transesterificación (Berry y Paterson, 1990). Dependiendo de su origen, las lipasas pueden tener un peso molecular variable entre 20000 y $200.000 \mathrm{Da}$, con actividad en amplios rangos de $\mathrm{pH}$ (4.0 a 9.0) y temperatura (ambiente hasta $70^{\circ} \mathrm{C}$ ) (Borgströn y Brockman, 1984). Con relación a su regioselectividad en substratos acilglicerólicos, pueden ser clasificadas en lipasas: 1,3 regioespecíficas, ácido graso específicas y no regio-específicas (Macrae y Hammond, 1987; Castro y Anderson, 1995).

Las lipasas han sido encontradas en muchos tejidos y fluidos de animales (mamíferos) y vegetales, o bien pueden ser producidas por procesos fermentativos utilizando diversas especies de microorganismos (hongos y bacterias). Actualmente, desde el punto de vista económico e industrial, los microorganismos han sido los preferidos como fuentes de lipasas (Mukherjee, 1994; Castro y Anderson, 1995).

Trabajos recientes demuestran que es posible obtener extractos parcialmente purificados de lipasas de plantas por técnicas relativamente simples a partir de materias primas de bajo costo (Mukherjee, 1994; Jachmanián et al., 1995).

En el caso del grano de arroz, que posee un potente sistema de lipasas, la mayor parte de esta actividad esta localizada en las capas externas del grano, o sea en el salvado. Este hecho convierte al salvado en una excelente materia prima para la obtención de lipasas, visto que se trata de un subproducto de bajo valor económico y de gran disponibilidad.

Las lipasas del salvado de arroz han sido estudiadas, principalmente con el propósito de determinar su mecanismo de acción, así como sus características físico-químicas (Funatsu et al., 1971, Aizono et al., 1971; 1973; 1976; Shastri y Raghavendra Rao, 1976, Fujiki et al., 1978; Takano, 1993).

Tres tipos principales de lipasas han sido descritas (Funatsu et al., 1971; Aizono et al., 1971; 1976). La lipasa I activada por iones $\mathrm{Ca}^{++}$a baja concentra- 
ción $\left(5 \times 10^{-4} \mathrm{M}\right)$. La lipasa II con peso molecular de 33000 $\mathrm{Da}$, formada de varias sub-unidades unidas por enlaces disulfuro (Fujiki et al., 1978). Ambas lipasas muestran actividad óptima a pH entre 7.5 y 8.0, hidrolizando triacilgliceroles de cadena corta o larga, incluyendo trioleína, aceites de arroz, oliva y coco, sin embargo, su actividad es muy superior sobre substratos de cadena corta $\left(\mathrm{C}_{2}-\mathrm{C}_{6}\right)$ (Borgströn y Brockman, 1984). Otros tipos de lipasas como fosfolipasas $A_{1}, A_{2}, B, C$ y $D$ (Takano, 1993), galactolipasas (Matsuda y Hirayama, 1979) y esterasas (Tsuzuki et al., 1994), también han sido aisladas a partir del salvado de arroz.

La obtención de extractos de lipasas crudos ha sido propuesta por diversos investigadores, donde tres métodos han sido descritos para este propósito (Funatsu et al., 1971; Lin y Hang, 1983; Hills y Mukherjee, 1990) y muestran diferencias considerables entre ellos, no solamente en el proceso (solución y condiciones de extracción) sino principalmente con respecto a su actividad y rendimiento. En este trabajo se realizó un estudio comparativo de los métodos para obtención de extractos crudo y semi-purificado de lipasas de salvado de arroz, con el objetivo de determinar su eficiencia.

\section{MATERIAL Y MÉTODOS}

El salvado de arroz, recién procesado, fue gentilmente cedido por «Máquina São José» Campinas, SP, Brasil. La composición centesimal del salvado fue determinada siguiendo los métodos: humedad (AACC, 1983 n. ${ }^{\circ} 44-19$ ), proteína bruta (AACC, 1983 n. ${ }^{\circ} 46-12$, factor 6.25), cenizas (AOAC, 1980 n. $^{\circ}$ 923.03), fibra bruta (AACC, 1983 n. ${ }^{\circ} 33-10$ ), extracto etéreo (AOAC, $\left.1984 \mathrm{n} .{ }^{\circ} 27.004\right)$ y acidez del aceite (AOCS, 1993 n. ${ }^{\circ}$ Ca 5a-40).

Obtención del extracto crudo: El salvado de arroz fue desengrasado en un extractor Soxhlet con éter de petróleo, en proporción salvado/solvente 1:6. El extracto crudo de lipasas fue obtenido utilizando los métodos descritos por: 1- Funatsu et al., (1971), con solución de $\mathrm{CaCl}_{2}$ 0.01M; 2- Lin y Huang (1983) con solución de sacarosa $0.6 \mathrm{M}, \mathrm{KCl} 10 \mathrm{mM}, \mathrm{MgCl}_{2}$ $1 \mathrm{mM}$, ditiotreitol $2 \mathrm{mM}$ y 3 - Hills y Mukherjee (1990) con solución de sacarosa $0.4 \mathrm{M}$, ditiotreitol $2 \mathrm{mM}$, usando en todos los casos una relación salvado desengrasado/solución de 1:8. Las variables estudiadas fueron: $\mathrm{pH}$ (6.0, 7.0 y 8.0) con tampones McLlvaine y tiempo de extracción (1h, $2 \mathrm{~h}$ y $3 \mathrm{~h}$ ) con agitación constante de $200 \mathrm{rpm}$ y temperatura de $4{ }^{\circ} \mathrm{C}$. Las suspensiones resultantes fueron centrifugadas a $5.000 \mathrm{rpm}$ durante 20 minutos a $4{ }^{\circ} \mathrm{C}$. Los sobrenadantes obtenidos (extracto crudo) fueron evaluados con respecto a su actividad lipasa.

Actividad enzimática: La actividad de la lipasa fue determinada utilizando el substrato sintético p-ni- trofenilaurato (Dodecanoic acid 4-nitrophenyl ester)(SIGMA). El medio de reacción consistía en 1.9 $\mathrm{mL}$ de reactivo (concentración de $0.847 \mathrm{mg} / \mathrm{mL}$ en solución de Mcllvaine $0.05 \mathrm{M}$ a pH $=8.0$ ) y $0.1 \mathrm{~mL}$ de extracto crudo (solución de enzima). El sistema de reacción fue inmediatamente incubado a $35^{\circ} \mathrm{C}$ por 30 minutos en baño agitador a 200 oscilaciones por minuto. La reacción fue interrumpida agregando $4 \mathrm{~mL}$ de acetona, seguido de centrifugación a $2000 \mathrm{rpm}$ durante 5 minutos. El sobrenadante fue separado y el p-nitrofenol liberado durante la reacción (de color amarillo) fue cuantificado en un espectrofotómetro a $410 \mathrm{~nm}$ con ayuda de una curva de calibrado (Palmiano y Juliano, 1973; Pastore, 1992).

Purificación del extracto crudo de lipasa: El salvado de arroz desengrasado (1 $\mathrm{kg})$ fue extraído con una solución de cloruro de calcio (4L) $0.01 \mathrm{M} \mathrm{a}$ $\mathrm{pH} 8.0$, y temperatura controlada a $4{ }^{\circ} \mathrm{C}$, con agitación constante de 200 oscilaciones durante un período de 2 horas. La suspensión fue centrifugada durante 20 minutos a $5000 \mathrm{rpm}$ y $4{ }^{\circ} \mathrm{C}$. El extracto crudo (sobrenadante) obtenido fue dividido en dos porciones tratadas como sigue:

a) Purificación con acetona (Ncube et al., 1993): Al extracto crudo fueron adicionadas pequeñas porciones de acetona a $-20{ }^{\circ} \mathrm{C}$ hasta alcanzar $60 \%(\mathrm{v} / \mathrm{v})$ con agitación constante durante 30 minutos en baño de hielo. El precipitado formado fue separado por centrifugación a $10.000 \mathrm{rpm}$ durante 15 minutos y temperatura de $4{ }^{\circ} \mathrm{C}$, y el exceso de acetona fue retirado por evaporación a presión reducida (Ncube et al., 1993). El extracto proteico semi-purificado (fracción acetona) fue pulverizado en un mortero.

b) Purificación con sulfato amónico (Funatsu et al., 1971): Al extracto crudo le fue adicionado sulfato amónico hasta una saturación del $60 \%$, con agitación constante. El precipitado formado fue separado por centrifugación (10.000 rpm x 15 minutos) y se le adicionó una cantidad mínima de solución de $\mathrm{CaCl}_{2}$ $0.001 \mathrm{M}$ seguido de diálisis a $4{ }^{\circ} \mathrm{C}$ por 48 horas. El extracto proteico semi-purificado (fracción sulfato amónico) fue liofilizado y pulverizado en un mortero.

Contenido proteico: El contenido proteico de los extractos semi-purificados fue determinado por el método de Lowry descrito en Kresze (1988), utilizando albúmina de suero bovino, como patrón proteico.

\section{RESULTADOS Y DISCUSIÓN}

\subsection{Composición centesimal del salvado de arroz}

Los resultados de composición obtenidos (Tabla I), fueron comparables a los valores encontrados por otros autores (Saunders, 1990; Luh et al., 1991). 
Tabla I

Composición centesimal del salvado de arroz

\begin{tabular}{lc}
\hline \multicolumn{1}{c}{ Componente } & Contenido $(\%)\left({ }^{*}\right)$ \\
\hline Humedad & 9.43 \\
Proteína & 16.32 \\
Extracto etéreo & 14.46 \\
Fibra bruta & 13.28 \\
Cenizas & 8.06 \\
\hline
\end{tabular}

(*) Media representativa de tres repeticiones.

\subsection{Obtención del extracto crudo}

\section{Efecto del pH}

En la Tabla II se muestra el efecto de diferentes valores de $\mathrm{pH}$ utilizados durante el proceso de extracción sobre la actividad hidrolítica de los extractos crudos de lipasa, obtenidos con las soluciones 1, 2 y 3 .

Las soluciones de extracción 1 y 3 , presentaron los mejores resultados de actividad lipasa a $\mathrm{pH}$ 8.0, mientras que el extracto preparado con la solución 2, mostró actividad máxima a $\mathrm{pH} 6.0$, aunque a pH 8.0 su actividad no es despreciable.

Tabla II

Efecto del pH y tiempo de extracción sobre la actividad hidrolítica de extractos crudos de salvado de arroz

\begin{tabular}{cccccc}
\hline \multirow{2}{*}{$\begin{array}{c}\text { Experimento } \\
\mathbf{n}^{\circ}\end{array}$} & $\mathrm{pH}$ & $\begin{array}{c}\text { Tiempo } \\
\text { (horas) }\end{array}$ & & & \multicolumn{3}{c}{ Actividad Lipasa (mU) (*) } \\
\cline { 5 - 6 } & & & Solución 1 & Solución 2 & Solución 3 \\
\hline 1 & 6,0 & 1 & 29 & 128 & 43 \\
2 & 6,0 & 2 & 134 & 141 & 50 \\
3 & 6,0 & 3 & 81 & 65 & 76 \\
4 & 7,0 & 1 & 106 & 94 & 81 \\
5 & 7,0 & 2 & 115 & 99 & 94 \\
6 & 7,0 & 3 & 71 & 74 & 115 \\
7 & 8,0 & 1 & 157 & 94 & 116 \\
8 & 8,0 & 2 & 178 & 132 & 144 \\
9 & 8,0 & 3 & 90 & 82 & 169 \\
\hline
\end{tabular}

(*) Media representativa de tres repeticiones.

$\mathrm{U}=1 \mu \mathrm{mol}$ de nitrofenol liberado por minuto por $\mathrm{mL}$ de extracto durante la reacción. $\mathrm{mU}=\mathrm{U} / 1000$

2.- Sacarosa $0.6 \mathrm{M}, \mathrm{KCl} 10 \mathrm{mM}, \mathrm{MgCl}_{2} 1 \mathrm{mM}$, ditiotreitol $2 \mathrm{mM}$ (Lin y Huang, 1983) y 3.- Sacarosa 0.4M, ditiotreitol $2 \mathrm{mM}$ ) (Hills y Mukherjee, 1990).

\section{Efecto del tiempo}

El efecto del tiempo de extracción sobre la actividad, también puede ser observado en la Tabla II. Las soluciones 1 y 2 presentaron mejores resultados en un tiempo de extracción de 2 horas, siendo que la solución 3 muestra una curva creciente de actividad, hasta la tercera hora en todos los valores de $\mathrm{pH}$ probados. La actividad máxima con la solución 3 fue alcanzada a pH 8.0, con un tiempo de extracción de 3 horas, pero este resultado de actividad no es superior al obtenido con la solución 1 a pH 8.0 y 2 horas de extracción.

Los resultados presentados en la Tabla II son bastante diferentes, hecho ya esperado, por tratarse de soluciones de composición distinta, consecuentemente, es lógico que respondan de modo diferente a las condiciones del medio. La solución de extracción 1 , fue la que presentó los mejores resultados de actividad lipasa, con máximo (178mU a pH 8.0 y tiempo de extracción de 2 horas, existiendo una diferencia significativa (5\%) con respecto a la solución 3 con 3 horas de extracción, donde la actividad lipasa fue de $169 \mathrm{mU}$. Funatsu et al., (1971), encontró resultados semejantes para pH 6.0, con el mismo tiempo (2 horas).

\subsection{Rendimiento de extracción y actividad lipasa}

En la Tabla III están resumidos los resultados de rendimiento, actividad hidrolítica total y especifica y contenido de proteína de los extractos obtenidos con la solución de $\mathrm{CaCl}_{2} 0.01 \mathrm{M}$. Se observa un aumento en la actividad específica, debido a los procesos de purificación, de 4.2 y 5.2 veces para los procesos con acetona y sulfato amónico respectivamente.

Los resultados de actividad específica de la lipasa para el extracto crudo y la fracción de sulfato amónico fueron similares a los publicados por Funatsu et al., (1971) y Aizono et al., (1976).

Tabla III

Contenido de proteína, actividad de lipasa total, específica y rendimiento del extracto bruto obtenido con una solución de $\mathrm{CaCl}_{2} 0,01 \mathrm{M}$ y de sus fracciones semipurificadas

\begin{tabular}{lcccc}
\hline Extracto & $\begin{array}{c}\text { Proteina Total } \\
(\mathbf{m g})\left({ }^{*}\right)\end{array}$ & $\begin{array}{c}\text { Actividad Total } \\
(\mathrm{U})\left(\left(^{*}\right)\right.\end{array}$ & $\begin{array}{c}\text { Actividad } \\
\text { Especifica } \\
(\mathrm{mU} / \mathrm{mg})\end{array}$ & $\begin{array}{c}\text { Rendimiento } \\
(\%)\end{array}$ \\
\hline Bruto & 73920,0 & 896,0 & 12,1 & 100 \\
Fracción Acetona & 14667,6 & 743,9 & 50,7 & 83 \\
Fracción Sulfato Amónico & 11146,8 & 707,8 & 63,5 & 79 \\
\hline
\end{tabular}

(*) Media representativa de tres repeticiones. $U=1 \mu$ mol de nitrofenol liberado por minuto por $\mathrm{mL}$ de extracto la reacción.
$\mathrm{mU}=\mathrm{U} / 1000$

De los resultados observados, se puede concluir que la manera más eficiente de producir un extracto semi-purificado de lipasas de salvado de arroz es utilizando una solución de $\mathrm{CaCl}_{2} 0.01 \mathrm{M}$ para la obtención del extracto crudo, seguido de su purifica- 
ción parcial con sulfato amónico. Cabe resaltar que la fracción acetona presento actividad específica inferior que a la de sulfato amónico, pero la técnica para su obtención es mas rápida y menos laboriosa, siendo una alternativa viable si otros factores, además de la actividad, son considerados en la obtención de extractos de lipasas para utilización como biocatalizadores en reacciones de hidrolisis y/o esterificación.

\section{BIBLIOGRAFÍA}

Aizono, Y., Funatsu, M., Hayashi, K., Inamasu, M. y Yamaguchi, M. (1971). — «Biochemical studies rice bran lipase-Part II».-Agric. Biol. Chem. 35, 1973-1979.

Aizono, Y., Funatsu, M., Sugano, M., Hayashi, K. y Fujiki, Y. (1973). - «Enzymatic properties of rice bran lipase».-Agric. Biol. Chem. 37, 2031-2036.

Aizono, Y., Funatsu, M., Fujiki, Y. y Watanabe, M. (1976).- «Purification and characterization of rice bran lipase II».-Agric. Biol. Chem. 40, 317-324.

AACC (1983).-Approved methods of the American Association of Cereal Chemists, 8th ed., St. Paul, MN.

AOAC (1980).-Official and Recommended Practices of the Association Official Analytical Chemists., Champaign.

AOAC (1984).-Official and Recommended Practices of the Association Official Analytical Chemists., Champaign.

AOCS (1993).-O Official and Recommended Practices of the American Oil Chemists Society. Champaign.

Berry, D.R. y Paterson, A. (1990).- - Enzymes in the Food Industry» en "Enzyme Chemistry - Impact and Applications" 2nd Ed., pp. 340-342.-C.J. Suckling (Ed.) Chapman and Hall, London.

Borgström, B. y Brockman, H.L. (1984).- «Lipases».Eselvier, Amsterdam.

Castro, H.F. y Anderson, W.A. (1995).- «Fine chemicals by biotransformation using lipases".-Química Nova 18, 544-554.

Fujiki, Y., Aizono, Y. y Funatsu, M. (1978).— «Chemical properties of major subunit of rice bran lipase".Agric. Biol. Chem. 42, 599-606.

Funatsu, M., Aizono, Y., Hayashi, K., Watanabe, M. y Eto, M. (1971).- «Biochemical studies on rice bran lipase Part l».-Agric. Biol. Chem. 35, 734-742.

Hills, M.J. y Mukherjee, K.D. (1990).— «Triacylglycerol lipase from rape (Brassica napus L.) suitable for biotechnological purposes".-Applied Biochemistry and Biotechnology $26,1-10$
IUPAC-IUB (1979).-Commission on nomenclature, Enzyme nomenclature Academic Press, NY, pp. 6-19.

Jachmanián, I., Grompone, M.A. y Mukherjee, K.D. (1995).- «Lipase from germinating rapeseed as biocatalyst in hidrolysis and esterification reactions" en «Proceedings of Latin American Congress and Exhibit on Fats \& Oils Processing, 25-28/09/1995".-D. Barrera-Arellano, M.A.B.R.D'Arce y L.A.G Gonçalves (Eds.)., Unicamp, Campinas.

Kresze, G. B. (1988).- «Methods for Protein Determination» en «Methods of Enzymatic Analysis» 3rd ed. Vol. 2.H. U. Bergmeyer (Ed.). - VCH Publishers, Weinhein.

Lin, Y. H. y Huang, A. H. C. (1983).—-Lipase in lipid bodies of cotyledons of rape and mustard seedling".-Arch. Biochem. Biophys. 225, 360-369.

Luh, B. S., Barber, S. y Barber, C. B. (1991).— «Rice Bran» vol. 2, 2nd pp. 313-362.-Van Nostrand Reinhold, New York.

Macrae, A. R. y Hammond, R. C. (1987).-—Present and future application of lipase». Biotechnology Letters, 9 601-604.

Matsuda, H. y Hirayama, O. (1979)._-_Purification of galactolipase from rice bran by affinity chromatography on palmitolated gauze».-Agric. Biol. Chem. 43, 463-469.

Mukherjee, K. D., (1994).- - «lant lipases and their application in lipid biotransformations".-Prog. Lipid Res. 33, 165-174.

Ncube, I., Adlercreutz, P. y Read, J. (1993).—«Purification of rape seedling lipase and its use in organic media".-Biotechnol. Appl. Biochem, 17, 327-336.

Palmiano, E.P. y Juliano, B.O. (1973).— «Change in the activity of some hydrolases, peroxidases and catalases in the rice seed during germination".-Plant. Physiol. 52, 274-277.

Pastore, G.M. (1992).-_ «rodução e caracterização bioquímica de monoacilglicerol lipase microbiana e aplicação de lipases na hidrólise e esterificação enzimática".- Tese, UNICAMP, Campinas.

Saunders, R.M. (1990). - «The properties of rice bran as a foodstuff».-Cereal Foods World 35, 632-636.

Shastry, B.S. y Raghavendra Rao, M. (1976).— «Chemical studies on rice bran lipase".-Cereal Chem. 53, 190.

Takano, K. (1993)._ «Mechanism of lipid hydrolysis in rice bran".-Cereal Foods World 38, 695-698.

Tsuzuki, W, Kasumimoto, H., Kobayashi, S. y Suzuki, T. (1994).- «Esterase activity and free fatty acid accumulation in the bran of selected rice cultivars".Cereal Chemistry. 71, 162-165.

Recibido: Enero 1998 Aceptado: Septiembre 1998 